\author{
Athina C. Tsili \\ Constantine Tsampoulas \\ Maria I. Argyropoulou \\ Iordanis Navrozoglou \\ Y. Alamanos \\ Evangellos Paraskevaidis \\ Stavros C. Efremidis
}

\section{Comparative evaluation of multidetector CT and MR imaging in the differentiation of adnexal masses: authors' reply}

Received: 29 December 2008

Accepted: 11 January 2009

Published online: 13 February 2009

(C) European Society of Radiology 2009

This reply refers to the Letter to the Editor available at doi:10.1007/s00330-008-1316-7

A. C. Tsili $(\bowtie) \cdot$ C. Tsampoulas Clinical Radiology,

University Hospital of Ioannina, Leoforos S. Niarchou, 45500, Pl. Pargis, 2 , Ioannina, 453 32, Greece e-mail: a tsili@yahoo.gr Tel.: +30-26-51070708

Fax: +30-26-51097862

M. I. Argyropoulou

Medical School,

University of Ioannina,

Leoforos Panepistimiou, 45110,

Ioannina, Greece

I. Navrozoglou $\cdot$ E. Paraskevaidis Gynecology \& Obstetrics,

University Hospital of Ioannina,

Leoforos S. Niarchou, 45500,

Ioannina, Greece

\section{Y. Alamanos}

Medical School, Hygiene and Public

Health, University of Ioannina,

Leoforos Panepistimiou, 45110,

Ioannina, Greece

S. C. Efremidis

Medical School, Department

of Radiology, University of Ioannina,

Leoforos Panepistimiou, 45110,

Ioannina, Greece
Sir,

We thank the authors of this letter for their thoughtful remarks.

We agree that the performance of unenhanced CT is necessary for the full characterization of adnexal masses, especially in 'differentiating enhancing lesion components from haemorrhage or mucinous content'. This could probably enable the correct characterization of one of the falsenegative cases with multidetector CT (MDCT) in our study, by detecting the enhancing element within a borderline ovarian tumour with haemorrhagic content [1]. This study was one of the first conducted on our 16-row MDCT system, and unenhanced CT was not performed due to concern about irradiation. The effective dose for the CT protocol used in this study was measured at $7 \mathrm{mSv}$, i.e. not different from that for conventional CT of the abdomen [2]. The protocol we use now includes unenhanced CT of the pelvis, covering the area from the iliac crests to the symphysis pubis, or the adnexal mass if larger, followed by enhanced $\mathrm{CT}$ of the abdomen and pelvis.

Regarding the area of coverage with MR imaging this included the pelvis 'covering the area from the iliac crests to the symphysis pubis, or the ovarian mass, if larger', as already mentioned in our paper in the 'Material and methods' section [1].
With regards to the statistical analysis, the ROC curve was produced using the SPSS/PC (version 15.0) statistical software. ROC curves for each diagnostic method were based on 67 patients, who underwent multidetector CT, MR imaging and histopathological examination, which was used as gold standard. The SPSS/PC (version 15.0) did not estimate statistical significance between two ROC curves. It just estimated the areas under the curve (0.921 and 0.982 for multidetector CT and MR imaging, respectively). We just compared these percentages, and the difference was not statistically significant for $N=67$.

\section{References}

1. Tsili AC, Tsampoulas C, Argyropoulou M, Navrozoglou I, Alamanos Y, Paraskevaidis E, Efremidis SC (2008) Comparative evaluation of multidetector CT and MR imaging in the differentiation of adnexal masses. Eur Radiol 18:1049-1057

2. Tsili AC, Tsampoulas C, Charisiadi A, Kalef-Ezra J, Dousias V, Paraskevaidis E, Efremidis SC (2008) Adnexal masses: accuracy of detection and differentiation with multidetector computed tomography. Gynecol Oncol 110:22-31 